\title{
Symptoms of depression but not anxiety are associated with central obesity and cardiovascular disease in people with type 2 diabetes: the Edinburgh Type 2 Diabetes Study
}

\author{
J. Labad • J. F. Price • M. W. J. Strachan • \\ F. G. R. Fowkes $\cdot$ J. Ding $•$ I. J. Deary $\cdot$ A. J. Lee $\cdot$ \\ B. M. Frier • J. R. Seckl • B. R. Walker • \\ R. M. Reynolds • \\ on behalf of the Edinburgh Type 2 Diabetes Study \\ Investigators
}

Received: 29 October 2009 / Accepted: 6 November 2009 /Published online: 10 December 2009

(C) Springer-Verlag 2009

\begin{abstract}
Aims/hypothesis The aim of the study was to identify risk factors for depression and anxiety in a well-characterised cohort of individuals with type 2 diabetes mellitus.

Methods We used baseline data from participants $(n=1,066$, $48.7 \%$ women, aged $67.9 \pm 4.2$ years) from the Edinburgh Type 2 Diabetes Study. Symptoms of anxiety and depression were assessed using the Hospital Anxiety and Depression Scale (HADS). Obesity was characterised according to both overall (body mass index, fat mass) and abdominal (waist circumference) measurements. Cardiovascular disease was assessed by questionnaire, physical examination and review of medical records. Stepwise multiple linear regression was performed to identify explanatory variables related to either anxiety or depression HADS scores.
\end{abstract}

Electronic supplementary material The online version of this article (doi:10.1007/s00125-009-1628-9) contains supplementary material, which is available to authorised users.

J. Labad·J. R. Seckl • B. R. Walker · R. M. Reynolds $(\bowtie)$

Endocrinology Unit, Centre for Cardiovascular Science,

Queen's Medical Research Institute, University of Edinburgh,

47 Little France Crescent,

Edinburgh EH16 4TJ, UK

e-mail: R.Reynolds@ed.ac.uk

J. F. Price $\cdot$ F. G. R. Fowkes $\cdot$ J. Ding

Centre for Population Health Sciences, University of Edinburgh,

Edinburgh, UK

J. F. Price $\cdot$ I. J. Deary $\cdot$ J. R. Seckl

Centre for Cognitive Ageing and Cognitive Epidemiology,

University of Edinburgh,

Edinburgh, UK
Results Abdominal obesity (waist circumference) and cardiovascular disease (ischaemic heart disease and anklebrachial pressure index) were related to depression but not anxiety. Lifetime history of severe hypoglycaemia was associated with anxiety. Other cardiovascular risk factors or microvascular complications were not related to either anxiety or depressive symptoms.

Conclusions/interpretation Depression but not anxiety is associated with abdominal obesity and cardiovascular disease in people with type 2 diabetes mellitus. This knowledge may help to identify depressive symptoms among patients with type 2 diabetes who are at greatest risk.

Keywords Anxiety · Depression · Diabetes · Obesity

M. W. J. Strachan

Metabolic Unit, Western General Hospital,

Edinburgh, UK

I. J. Deary

Department of Psychology, University of Edinburgh,

Edinburgh, UK

A. J. Lee

Section of Population Health, University of Aberdeen,

Aberdeen, UK

B. M. Frier

Department of Diabetes, Royal Infirmary of Edinburgh,

Edinburgh, UK 


\author{
Abbreviations \\ ABPI Ankle-brachial pressure index \\ ACR Albumin to creatinine ratio \\ ET2DS Edinburgh Type 2 Diabetes Study \\ HADS Hospital Anxiety and Depression Scale \\ LDR Lothian Diabetes Register
}

\section{Introduction}

Although it is recognised that people with type 2 diabetes mellitus often have anxiety and depression [1, 2], the potential risk factors for these conditions have not been well defined. Most studies examining psychological disorders occurring in type 2 diabetes have focused on depression, which has been associated with poorer glycaemic control [3], more metabolic complications [3, 4] including ischaemic heart disease [2, 4], and greater mortality [5]. The poorer prognosis in patients with depression may in part be explained by a lower adherence to medications or dietary recommendations and a higher prevalence of obesity [4] and of other cardiovascular risk factors [4]. Symptoms of anxiety are often reported by people with type 2 diabetes [1], and with depression or obesity [6]. However, it is not known whether anxiety symptoms are related to obesity or cardiovascular risk in type 2 diabetes.

Previous studies [3, 5] exploring the relationship between depression and the metabolic profile in type 2 diabetes have assessed adiposity by body mass index but more detailed assessments such as waist circumference or body fat distribution are lacking. The differentiation between central and overall obesity when assessing depression is important as recent studies suggest that central (rather than overall) obesity is associated with depression in non-diabetic populations [7] and also with an adverse metabolic profile [8].

We therefore aimed to identify risk factors for depression and anxiety in a large and very well-characterised sample of people with type 2 diabetes. We controlled for both overall (BMI and fat mass) and abdominal obesity (waist circumference) measures.

\section{Methods}

The Edinburgh Type 2 Diabetes Study (ET2DS) is a population-based prospective cohort study designed to determine the association between potentially modifiable risk factors and cognitive decline in people with type 2 diabetes. Ethical permission was obtained from the Lothian Research Ethics Committee and written informed consent was obtained from all participants. The complete protocol is described elsewhere [9]. In brief, individuals with type 2 diabetes (WHO criteria) living in Lothian, Scotland, were selected from the Lothian Diabetes Register (LDR). People aged between 60 and 74 years on 1 August 2006 were selected by sex and 5 year age bands from computerrandomised lists of eligible individuals from the LDR. After exclusion of patients with pre-defined criteria [9], 1,066 people were willing and eligible to take part in the ET2DS.

Clinical assessment Participants completed a questionnaire at baseline to assess socio-demographic and lifestyle variables, and clinical information related to their diabetes and cardiovascular status, including questions on medical diagnoses and/or treatment for angina, myocardial infarction, stroke, hypertension and hypercholesterolaemia. Lifetime history of severe hypoglycaemic episodes was defined by self-report as the presence of at least one hypoglycaemic episode needing assistance by another person. Details on the year of cardiovascular diagnosis or event, and hospital or general practice attended were collected to enable further validation of diagnoses after comparison with data from the LDR and from the Information Service Division (Scotland's national organisation for health information, statistics and information technology services). The Hospital Anxiety and Depression Scale (HADS) was used to evaluate current anxiety and depressive symptoms. This scale performs well in screening for the separate dimensions of anxiety and depression in patients from non-psychiatric hospital clinics [10].

A complete physical examination was performed including measurement of systolic and diastolic brachial blood pressures, waist circumference and body fat percentage by bio-electrical impedance. As the maximum percentage fat mass that could be recorded was $50 \%$, we categorised this variable into quartiles, using different cut-off points for men and women because of the known sex differences in fatmass distribution [7]. A resting 12 lead electrocardiogram was recorded. Assessment of ankle-brachial pressure index (ABPI), neuropathy and retinopathy, as well as further definitions of cardiovascular disease, cardiovascular risk factors and metabolic complications, are detailed in Electronic Supplementary Material (ESM) Table 1.

Blood and urine samples Venous blood samples were taken after an overnight fast for measurement of $\mathrm{HbA}_{1 \mathrm{c}}$, and plasma total and HDL-cholesterol. An early-morning specimen of urine was obtained to calculate the albuminto-creatinine ratio (ACR).

Statistical analyses Data were analysed using SPSS 15.0. As HADS anxiety and depression scores were skewed, a square-root transformation was applied. Multiple linear 
regression was performed to identify explanatory variables related to either anxiety or depression scores (used as continuous variables). Sex was forced to enter the equation in the first step (so all models are adjusted for sex) whereas other independent variables were tested with a forward stepwise procedure: age; employment status; education level; marital status; anxiety or depression HADS score; obesity-related variables (BMI, waist circumference and fat mass); duration of diabetes mellitus; history of severe hypoglycaemia; diabetes treatment; $\mathrm{HbA}_{1 \mathrm{c}}$; smoking; alcohol consumption; ischaemic heart disease; stroke; ABPI; ACR; neuropathy; retinopathy; hypertension; dyslipidaemia; and treatment with thyroxine, antidepressants or glucocorticoids. Interactions between sex and independent variables were tested and significant interactions were included in the final equations. Two analyses were performed, using the square-root transformed HADS scores for anxiety (analysis 1) or depression (analysis 2) as the dependent variable.

\section{Results}

Clinical characteristics and metabolic complications of the participants are described in Table 1. Results from the multiple linear regression models used to select explanatory variables related to anxiety and depression HADS scores are shown in Table 2. HADS depressive scores were related to anxiety scores. Abdominal obesity and ischaemic heart disease were positively related to depression but not to anxiety. Lower ABPI measurements were also associated with depression. Treatment with insulin was positively related to depression and inversely related to anxiety. History of severe hypoglycaemia was associated with anxiety. Individuals living with a partner reported less anxiety and those with a lower education reported more depression. Female participants and those taking antidepressants reported more anxiety symptoms. There was however, a sex interaction effect in relation to the antidepressant treatment (i.e. women taking antidepressants reported less anxiety). No significant sex interaction effects were found in the depression regression model.

\section{Discussion}

This is the largest study to investigate the factors predicting symptoms of anxiety and depression in a well-characterised cohort of people with type 2 diabetes. We found a positive association between abdominal obesity and depression but not anxiety. Cardiovascular disease measures (ischaemic heart disease and lower ABPI) were related only to depression.
Table 1 Baseline clinical variables in the ET2DS $(n=1,066)$

\begin{tabular}{|c|c|}
\hline Variable & $\begin{array}{l}\text { Mean (SD) } \\
\text { or } n(\%)\end{array}$ \\
\hline \multicolumn{2}{|l|}{ Socio-demographic/lifestyle } \\
\hline Female sex & $519(48.7)$ \\
\hline Age at assessment (years) & $67.9(4.2)$ \\
\hline \multicolumn{2}{|l|}{ Current marital status } \\
\hline Married & $744(69.9)$ \\
\hline Living with long-term partner & $54(5.1)$ \\
\hline Single & $159(14.9)$ \\
\hline Widowed & $107(10.1)$ \\
\hline \multicolumn{2}{|l|}{ Education (highest level completed) } \\
\hline University/college & $171(16.0)$ \\
\hline Other professional qualification & $307(28.8)$ \\
\hline Primary or secondary school & $588(55.2)$ \\
\hline \multicolumn{2}{|l|}{ Current employment status } \\
\hline Worker & $152(14.3)$ \\
\hline Retired & $864(81.1)$ \\
\hline Other: housewife, unemployed & $50(4.7)$ \\
\hline \multicolumn{2}{|l|}{ Ethnic group } \\
\hline White & $1,016(95.3)$ \\
\hline Other & $50(4.7)$ \\
\hline Current smoker & $148(13.9)$ \\
\hline \multicolumn{2}{|l|}{$\begin{array}{l}\text { Alcohol consumption in preceding year } \\
\text { (frequency) }\end{array}$} \\
\hline Never & $218(20.6)$ \\
\hline 1-4 drinks per month & $463(43.7)$ \\
\hline 2-5 drinks per week & $268(25.3)$ \\
\hline 6 or more drinks per week & $110(10.4)$ \\
\hline \multicolumn{2}{|l|}{ Diabetes mellitus } \\
\hline Duration (years) & $9.1(6.5)$ \\
\hline $\mathrm{HbA}_{1 \mathrm{c}}$ & $7.4(1.1)$ \\
\hline \multicolumn{2}{|l|}{ Treatment } \\
\hline Diet alone & $201(18.9)$ \\
\hline Oral hypoglycaemic agents & $678(63.6)$ \\
\hline Insulin \pm oral hypoglycaemic agents & $187(17.5)$ \\
\hline Lifetime history of severe hypoglycaemic & $113(10.8)$ \\
\hline
\end{tabular}

episodes (1 or more)

Cardiovascular and other metabolic complications

Systolic blood pressure $(\mathrm{mmHg})$

$133.3(16.4)$

Diastolic blood pressure $(\mathrm{mmHg})$

$69.1(9.0)$

Hypertension

956 (89.7)

HDL-C (mmol/l)

$1.3(0.4)$

Dyslipidaemia

$923(86.6)$

Ischaemic heart disease (angina or

$330(31.0)$

myocardial infarction)

Myocardial infarction

$150(14.1)$

Angina (lifetime history)

$298(28.0)$

Cerebrovascular disease (stroke or TIA)

$93(8.7)$

ABPI

$0.981(0.207)$

Neuropathy

$520(48.8)$

ACR (mg/mmol) 
Table 1 (continued)

\begin{tabular}{lc}
\hline Variable & $\begin{array}{l}\text { Mean (SD) } \\
\text { or } n(\%)\end{array}$ \\
\hline $\begin{array}{l}\text { Retinopathy } \\
\text { Anthropometric measures }\end{array}$ & $400(37.5)$ \\
Weight $(\mathrm{kg})$ & $86.5(16.2)$ \\
BMI $\left(\mathrm{kg} / \mathrm{m}^{2}\right)$ & $31.4(5.7)$ \\
Waist $(\mathrm{cm})$ & \\
$\quad$ Men & $108.2(12.1)$ \\
$\quad$ Women & $105.5(13.5)$ \\
Mood scores & \\
HADS-anxiety score & $5.7(3.9)$ \\
HADS-depression score & $3.9(2.9)$ \\
Treatments & \\
Taking antidepressant & $137(12.9)$ \\
Taking glucocorticoid & $147(13.8)$ \\
Taking thyroxine & $121(11.4)$ \\
\hline
\end{tabular}

Sample size differs for some variables with $<3 \%$ missing data

TIA, transient ischaemic attack

Our data support an association between depression and obesity in type 2 diabetes, as has been suggested by earlier studies in both non-diabetic [6] and diabetic [3] populations. When three obesity measures (BMI, fat mass and waist circumference) were examined in the multivariate analysis, only waist circumference was significantly related to depression. These findings are in accord with a recent prospective study [7] in a non-diabetic population in which visceral obesity as determined by computed tomography (but not BMI or fat mass) was related to the incidence of depression. However, the effect size was small, explaining only $4 \%$ of the variability of the data in the final regression model for depressive symptoms.

Although it is possible that the relationship between visceral obesity, cardiovascular disease and depression could be explained by differences in cardiovascular risk factors, we found that cardiovascular risk factors were not related to either anxiety or depressive symptoms, albeit that the lack of association with these cardiovascular risk factors may be obscured by treatment effects. In contrast to previous studies in type 2 diabetes which have reported associations between depression and metabolic complications, including coronary heart disease, only in men [3], no significant sex differences were evident in our study. Interestingly, we found a positive relationship between lifetime history of severe hypoglycaemia and symptoms of anxiety. It is not known whether anxiety per se impairs awareness of hypoglycaemia or interferes with the ability to self-treat a fall in blood glucose, or both.

The main limitation of our study is the cross-sectional design and so a causal relationship between clinical variables and anxiety or depressive symptoms cannot be inferred. Although we did not include a non-diabetic comparison group, we included the full spectrum of people type 2 diabetes ranging from those using dietary therapy

Table 2 Significant explanatory variables included in the final model for each multiple linear regression analysis testing anxiety or depression score in male and female patients

\begin{tabular}{|c|c|c|c|c|c|}
\hline Variable & $R^{2}$ change & $\beta$ & SE & $95 \% \mathrm{CI}$ & $p$ value \\
\hline \multicolumn{6}{|l|}{ Analysis 1 (anxiety) } \\
\hline Female sex & 0.067 & 0.407 & 0.052 & $0.305,0.509$ & $<0.001$ \\
\hline HADS-depression $^{\mathrm{a}}$ & 0.263 & 0.595 & 0.032 & $0.532,0.657$ & $<0.001$ \\
\hline Lifetime history of severe hypoglycaemia & 0.008 & 0.293 & 0.082 & $0.133,0.453$ & $<0.001$ \\
\hline Marital status (living with partner) & 0.005 & -0.299 & 0.116 & $-0.526,-0.071$ & 0.010 \\
\hline Taking antidepressant treatment & 0.004 & 0.399 & 0.114 & $0.176,0.622$ & $<0.001$ \\
\hline Treatment with insulin $( \pm$ OHA) & 0.004 & -0.167 & 0.068 & $-0.300,-0.033$ & 0.014 \\
\hline Interaction female sex by antidepressant treatment & 0.004 & -0.367 & 0.147 & $-0.656,-0.078$ & 0.013 \\
\hline \multicolumn{6}{|l|}{ Analysis 2 (depression) } \\
\hline Female sex & 0.012 & -0.002 & 0.043 & $-0.085,0.082$ & 0.971 \\
\hline HADS-anxiety ${ }^{a}$ & 0.279 & 0.427 & 0.023 & $0.383,0.471$ & $<0.001$ \\
\hline Waist circumference (in $\mathrm{cm}$ ) & 0.038 & 0.010 & 0.002 & $0.007,0.014$ & $<0.001$ \\
\hline Ischaemic heart disease & 0.023 & 0.241 & 0.045 & $0.153,0.329$ & $<0.001$ \\
\hline Treatment with insulin $( \pm$ OHA) & 0.007 & 0.179 & 0.055 & $0.071,0.286$ & 0.001 \\
\hline Low education (primary or secondary school) & 0.003 & 0.087 & 0.041 & $0.006,0.167$ & 0.035 \\
\hline ABPI & 0.003 & -0.208 & 0.099 & $-0.402,-0.013$ & 0.036 \\
\hline
\end{tabular}

OHA, oral hypoglycaemic agent 
alone to insulin-treated individuals with complications. We used a psychometric scale for assessing anxiety and depression rather than a structured interview for mental disorders, thus clinical diagnoses of anxiety or depressive disorders could not be made and information about current or prior history of depressive disorders was not available. As symptoms of anxiety and depression may be obscured by antidepressant treatment, we repeated all regression analysis after excluding those participants taking antidepressants; the final results did not change.

In summary, our results suggest that depression but not anxiety is associated with abdominal obesity and cardiovascular disease in people with type 2 diabetes. This relationship is independent of cardiovascular risk factors. Anxiety is also associated with risk of hypoglycaemia. In the clinical setting, knowledge of these risk factors may help to identify depressive symptoms among people with type 2 diabetes.

Acknowledgements This study was supported by a grant from the Medical Research Council. We thank the participants and staff of the Edinburgh Type 2 Diabetes Study (particularly J. McKnight and P. Halpin for retinopathy grading) and the staff at the Wellcome Trust Clinical Research Facility where the study was performed. We are also grateful for assistance from our ET2DS collaborators who contributed to the study design and collected data (K. Swa and G. D. O. Lowe).

\section{References}

1. Hermanns N, Kulzer B, Krichbaum M, Kubiak T, Haak T (2005) Affective and anxiety disorders in a German sample of diabetic patients: prevalence, comorbidity and risk factors. Diabet Med 22:293-300

2. Adriaanse MC, Dekker JM, Heine RJ et al (2008) Symptoms of depression in people with impaired glucose metabolism or type 2 diabetes mellitus: the Hoorn Study. Diabet Med 25:843-849

3. Katon W, von Korff M, Ciechanowski P et al (2004) Behavioral and clinical factors associated with depression among individuals with diabetes. Diabetes Care 27:914-920

4. Bruce DG, Davis WA, Starkstein SE, Davis TM (2005) A prospective study of depression and mortality in patients with type 2 diabetes: the Fremantle Diabetes Study. Diabetologia 48:2532-2539

5. Katon WJ, Rutter C, Simon G et al (2005) The association of comorbid depression with mortality in patients with type 2 diabetes. Diabetes Care 28:2668-2672

6. Kasen S, Cohen P, Chen H, Must A (2008) Obesity and psychopathology in women: a three decade prospective study. Int J Obes (Lond) 32:558-566

7. Vogelzangs N, Kritchevsky SB, Beekman AT et al (2008) Depressive symptoms and change in abdominal obesity in older persons. Arch Gen Psychiatry 65:1386-1393

8. Skilton MR, Moulin P, Terra JL, Bonnet F (2007) Associations between anxiety, depression, and the metabolic syndrome. Biol Psychiatry 62:1251-1257

9. Price JF, Reynolds RM, Mitchell RJ et al (2008) The Edinburgh Type 2 Diabetes Study: study protocol. BMC Endocr Disord 8:18

10. Bjelland I, Dahl AA, Haug TT, Neckelmann D (2002) The validity of the Hospital anxiety and depression scale. An updated literature review. J Psychosom Res 52:69-77 